\title{
Norois
}

Environnement, aménagement, société

\section{Le SCoT, instrument de gouvernance territoriale? La conduite locale de la concertation dans le Pays du Grand Clermont}

The SCOT, a tool for territorial governance? Local dialogue around the Pays du Grand Clermont

Salma Loudiyi

\section{OpenEdition}

Journals

\section{Édition électronique}

URL : https://journals.openedition.org/norois/2615

DOI : $10.4000 /$ norois. 2615

ISBN : 978-2-7535-1557-4

ISSN : 1760-8546

Éditeur

Presses universitaires de Rennes

\section{Édition imprimée}

Date de publication : 15 décembre 2008

Pagination : 37-56

ISBN : 978-2-7535-0805-7

ISSN : 0029-182X

Référence électronique

Salma Loudiyi, « Le SCoT, instrument de gouvernance territoriale? La conduite locale de la concertation dans le Pays du Grand Clermont », Norois [En ligne], 209 | 2008/4, mis en ligne le 01 mars 2011, consulté le 14 janvier 2022. URL : http://journals.openedition.org/norois/2615 ; DOI : https:// doi.org/10.4000/norois. 2615 


\title{
LE SCOT, INSTRUMENT DE GOUVERNANCE TERRITORIALE? \\ LA CONDUITE LOCALE DE LA CONCERTATION \\ dans le Pays du Grand Clermont
}

\author{
SALMA LOUDIYI \\ UMR METAFORT INRA \\ (INRA/Agro Paris Tech - ENGREF), \\ Domaine des Cézeaux, BP 90054, 24 Avenue des Landais - 63171 Aubières cedex 9 \\ salma.loudiyi@agroparistech.fr
}

\begin{abstract}
RÉSUMÉ
L'analyse d'un dispositif de gouvernance territoriale est présentée à travers l'élaboration du SCoT du Grand Clermont, instrument de planification stratégique réunissant les acteurs de l'agglomération clermontoise et de ses périphéries. L'article décrit le contexte clermontois et la conduite locale de la concertation autour du diagnostic du SCoT. L'auteur analyse le déroulement du processus de concertation en analysant les effets propres et induits par l'instrumentation du SCoT.
\end{abstract}

MOTS CLÉ : planification stratégique - gouvernance - SCoT-participation-outils de concertation - agglomération clermontoise

\footnotetext{
ABSTRACT

The SCoT, a tool for territorial governance? Local dialogue around the Pays du Grand Clermont

To grasp a governance device, the author suggest an analysis of the SCoT drawing up in Clermont-Ferrand (France). The SCoT is a tool for strategic planning which set terms of participation of a large set of stakeholders. The paper describe the local context and the dialogue process applied to carry out the diagnosis step of the SCoT. The author describe the dialogue process by emphasising its own and led effects.
}

KEY WORDS : strategic planning - governance-SCoT-participation-dialogue tools - Urban region of Clermont-Ferrand

La transformation et le renouvellement de l'action publique induits par le désengagement de l'État, la décentralisation des pouvoirs et la montée des incertitudes à propos de l'avenir des territoires laissent place à de nouvelles conceptions des modes d'action publique. À une action publique régalienne se substitue une action publique partenariale dont le développement des politiques contractuelles et les démarches de projet sont des illustrations. Le projet est ainsi considéré 
comme un instrument d'action publique et renvoie à de nouvelles modalités d'intervention qui intègrent le principe d'une participation plus large d'acteurs dans la définition des projets d'aménagement et de développement des territoires. À des instruments d'action publique tirés du savoir expert et agissant sur une réalité sociale perçue comme mesurable et des problèmes bien identifiés, succèdent des instruments d'action publique, insérés dans un monde incertain (Callon et al., 2001) et accompagnant la construction collective de connaissances et de réponses aux enjeux territoriaux. L'action publique s'appuie sur des référentiels puisés dans les modèles de la nouvelle gouvernance (Fergusson, 2008) qui constituent une rupture paradigmatique dans la conduite des politiques publiques. Une participation plus large est conçue comme autant de nouvelles ressources mobilisables dans la conception des territoires et comme réponse circonstanciée à des problématiques pour lesquelles le savoir expert n'est plus le seul invoqué. Les projets appellent ainsi à la mise en œuvre d'une action collective visant la construction d'un consensus entre une pluralité d'acteurs et suit, pour cela, une rationalité interactionniste et processuelle (Pinson, 2004). Ces démarches passent par la mise en place de dispositifs d'échanges et d'interaction, dont on attend généralement qu'ils produisent une vision partagée et commune des normes et des règles d'action, qu'ils fassent émerger des accords sur les stratégies de développement à mettre en œuvre et qu'ils renforcent l'action collective et les réseaux d'acteurs.

Cependant, la participation d'une pluralité d'acteurs dans le cadre de projets recouvre une grande variété de procédures, dont les objectifs peuvent aller du simple recueil de l'avis des acteurs concernés à une implication active dans la prise de décision. De la consultation à la concertation, ces nouvelles formes participatives concourent à l'évolution progressive de la mise en œuvre des politiques publiques et des nouveaux référentiels de la gouvernance. En effet, les dispositifs participatifs sont devenus aujourd'hui des modes de conduite de projet de territoire ou de planification stratégique. Ils engagent de nouvelles modalités de gouvernance territoriale dans la mesure où ils impliquent une participation d'un ensemble plus large d'acteurs dans la construction d'une vision collective du territoire de développement et la définition des stratégies territoriales. Mais dans la diffusion de ces façons de faire, les processus de coordination et de mise en œuvre de l'action collective passent par une appropriation locale des procédures et des modalités de gouvernance territoriale associées. Si les textes législatifs offrent la possibilité d'existence d'espaces et d'institutions qui permettent à de nouveaux acteurs (citoyens, groupements communautaires, associations, collectivités territoriales, etc.) une implication plus active dans la gestion des problématiques territoriales, force est de constater que les démarches de projets et leurs modes de régulation varient beaucoup suivant les situations locales.

Dans le cadre de cet article, nous proposons de questionner l'effectivité de la transformation de l'action publique en analysant la conduite de la concertation locale dans la mise en place d'un Schéma de Cohérence Territoriale (SCoT). Instrument d'action publique de nature générique, mis en œuvre par la loi Solidarité et renouvellement urbain (2000) et repris par la loi Habitat et urbanisme (2003), il renouvelle la conception de la planification urbaine dans la mesure où les enjeux proprement spatiaux laissent place à des enjeux de mobilisation sociale où la participation est une notion cardinale.

Nous aborderons cette question à travers une entrée analytique, l'instrumentation de l'action publique, qui permet d'appréhender les caractéristiques de la régulation et les modalités de la gouvernance territoriale mises en œuvre. Suivant P. Lascoumes et P. Le Galès (2004), il est entendu par l'instrumentation de l'action publique « l'ensemble des problèmes posés par le choix et l'usage d'outils (des techniques, des moyens d'opérer, des dispositifs) qui permettent de matérialiser et d'opérationnaliser l'action gouvernementale ». Autrement dit, cette entrée permet d'appréhender non seulement les caractéristiques des instruments de l'action publique mais également les conditions de leurs choix et les effets que génèrent ces choix. Les choix retenus dans la conduite de ce processus de planification urbaine induiraient des transformations de la construction des ressources et des territoires mais également de l'action collective organisée. 
Comme instrument de gouvernance territoriale, le SCoT structure et organise une action collective, produit par là des effets propres en même temps qu'il induit des effets latéraux. Nous proposons dans le cadre de cet article de les saisir à travers l'observation et le suivi de la phase de concertation visant la construction d'un diagnostic partagé du SCoT du Pays du Grand Clermont. Cette lecture s'attache à préciser le contexte local, les formes prises par la conduite locale de la concertation et plus précisément le choix des procédures mobilisées. Nous proposons de montrer comment l'instrumentation de l'action publique produit autant des effets propres que des effets induits à travers le choix des procédures.

Nous reviendrons dans une première partie sur le cadre analytique proposé autour de l'instrumentation de l'action publique et sur les opérations de planification concertée qui induisent des formes de gouvernance territoriale. Dans cette partie, nous montrerons que le SCoT présente les caractéristiques d'un instrument de gouvernance territoriale. La seconde partie est consacrée à l'analyse du cas clermontois. Nous retraçons le suivi de la conduite du diagnostic partagé du SCoT et nous mettons en lumière les choix retenus dans la conduite de la concertation et ses liens étroits avec le contexte local. Les procédures retenues et les outils mobilisés dans la conduite locale de la concertation orientent le regard vers les effets produits par l'instrumentation de l'action publique analysés dans la troisième partie.

\section{Instrumentation de l'action publique, SCoT et gouvernance territoriale}

\section{INSTRUMENTATION DES POLITIQUES PUBLIQUES}

Un instrument de politique publique est « un dispositif à la fois technique et social qui organise des rapports sociaux spécifiques entre la puissance publique et ses destinataires en fonction de représentations et des significations dont il est porteur» (Lascoumes et Le Galès, 2004). Suivant les auteurs, on peut dire que c'est un dispositif technique dans la mesure où un ensemble de techniques est mis en œuvre, utilisé et manipulé pour atteindre une finalité. C'est aussi un dispositif social parce qu'il permet de mettre en relation, constamment, une somme d'informations et un ensemble d'acteurs qui les réinterprètent régulièrement et collectivement; il est ainsi l'occasion de débats autour des questions qu'il convoque et des finalités qu'il vise. Sa vocation générique relève de sa possible application à des problèmes sectoriels divers et de sa capacité à gérer la transversalité des questions et des enjeux relatifs aux objets saisis par la collectivité.

Plusieurs instruments structurent l'action publique depuis des décennies en France. Chaque instrument a sa propre histoire et ses propriétés sont indissociables des finalités qui lui sont attribuées. Concernant les projets urbains qui nous intéressent plus précisément ici, G. Pinson (2004) souligne qu'en tant qu'instrument d'action publique, le projet urbain est doté de trois propriétés : il poursuit un objectif de mobilisation sociale, suivant la mise en œuvre d'une logique interactionniste et processuelle et a vocation d'affirmer des identités d'action en pérennisant des groupes d'acteurs solidarisés par la poursuite des mêmes objectifs. D'abord, le projet urbain est une activité proprement politique qui suppose la participation des acteurs du territoire concerné et la valorisation de ses ressources. La mobilisation sociale s'inscrit comme un nouveau référentiel de l'action publique et permet l'intégration et l'articulation de nouvelles ressources locales, qu'elles soient matérielles ou symboliques pour atteindre des objectifs construits collectivement. Elle s'appuie, en cela, sur des formes d'action innovantes dans la mesure où elle s'ancre dans de nouvelles formes de gouvernance. Pour G. Pinson (2004), la mobilisation sociale et l'articulation des ressources locales deviennent des enjeux centraux de l'action publique puisqu'ils déterminent les objectifs mêmes à atteindre par le collectif des acteurs engagés dans le processus de projet. Ensuite, la mobilisation sociale vise la construction graduelle d'un consensus opératoire autour des objectifs partagés entre acteurs engagés dans ce processus. Ces démarches passent par la mise en place de dispositifs d'échanges et d'interaction qui facilitent la construction d'un consensus et dont on attend la construction d'une vision partagée de la ressource commune, l'émergence de normes 
et de règles et la stabilisation progressive d'une identité d'action. Ces démarches peuvent prendre des formes diverses comme les dispositifs de concertation, où des stratégies d'aménagement et de développement peuvent émerger au terme de processus d'échanges entre une pluralité d'acteurs. Plus encore, la mobilisation sociale et les processus d'interaction construisant les accords sont censés assurer, par leur itération, une pérennisation des dispositions à la coordination et la coopération. La stabilisation des systèmes d'acteurs autour d'un projet apparaît ainsi plus importante que l'action en elle-même ou son contenu. Enfin, la démarche de construction collective d'un projet est éminemment politique dans la mesure où sa vocation est aussi de rendre visible un acteur collectif et de le positionner au sein d'un environnement souvent concurrentiel. La construction d'une intention politique et d'une identité collective permet ainsi de donner consistance à un territoire et la constitution d'un acteur collectif reconnu.

\section{LE SCOT, Un INSTRUMENT D’ACTION PUBLIQUE}

L'élaboration des schémas de cohérence territoriale (SCoT) apparaît aujourd'hui comme un nouvel instrument d'action publique structurant la planification stratégique à l'échelle urbaine et métropolitaine. Cet instrument renouvelle la conception de la planification naguère ancrée dans une dimension purement technique, où les enjeux spatiaux prédominaient, en y introduisant une vision plus stratégique relative à la prospective ${ }^{1}$, à la mise en cohérence des politiques publiques et à leur articulation territoriale. L'ambition nouvelle des SCoT vise une gestion économe des espaces, une mobilisation sociale contre les phénomènes ségrégatifs et une implication nouvelle dans le traitement collectif des enjeux de durabilité des systèmes territoriaux (Helluin, 2005).

Le SCoT s'inscrit dans une continuité de l'action publique et sa pérennisation à travers de nouveaux référentiels d'action. Il renouvelle la conception des schémas directeurs, où désormais, la démarche de projet prime avant tout. La mise en cohérence des politiques publiques sectorielles, la vision prospective, l'obligation du suivi à travers l'évaluation, rejoignent des objectifs de mobilisation sociale autour du projet, de choix territoriaux retenus pour sa mise en œuvre, de construction d'une vision partagée des ressources collectives et de pérennisation de l'action collective organisée. Établi sur un vaste périmètre, le SCoT est un instrument qui peut viser un objectif de complémentarité vis-à-vis d'une démarche de pays ou d'agglomération, en donnant un caractère prescriptif aux orientations de la charte de pays, ou du projet de l'agglomération, et en renforcer les programmes d'actions. Ces orientations peuvent être intégrées dans des objectifs spatialisés qui leur donnent un caractère opérationnel. Dispositif de nature générique, il s'accorde avec les spécificités des contextes locaux. L'état des lieux des démarches engagées en France métropolitaine depuis la promulgation de la loi SRU en témoigne. Si les SCoT visaient à institutionnaliser des espaces fonctionnels et au premier chef, les aires urbaines (définition INSEE), les choix retenus par les acteurs varient : seulement $31 \%$ d'entre eux couvrent un territoire plus large qu'une aire urbaine (CERTU, 2008) et 17 \% offrent une adéquation entre périmètre de Pays et SCoT (ETD, 2007).

Le SCoT est ainsi une démarche volontaire de la part d'un collectif d'acteurs réunis autour d'un espace d'action. Suivant la législation, il est élaboré à l’initiative des communes et de leurs groupements compétents sur un périmètre défini, qui peut concerner autant une EPCI qu'un groupement d’EPCI et de communes (Art. L. 122-3 de la loi SRU). La participation de collectivités territoriales ou de personnes publiques supplémentaires a évolué. L'article L. 121-4 a ainsi élargi l'association, à leur demande, aux organismes de gestion des PNR et à ceux compétents en matière d'organisation des transports urbains, à l'État, au département, à la région et aux chambres consulaires. Dans la même lignée mais suivant des modalités autres, les services déconcentrés de l'État sont associés au processus à la demande du préfet ou du président de l'EPCI. La gamme des acteurs consultés se trouve encore plus élargie avec le recueil de l'avis de tout organisme compétent en

1. C'est un document d'urbanisme qui doit définir les modalités de développement et d'aménagement d'un territoire pour un horizon de 15 ans. 
matière de politiques publiques en lien avec le SCoT ${ }^{2}$. De même, les objectifs de consultation visent une intégration territoriale « idéale » avec l'association de toute collectivité territoriale d'un État limitrophe pour inscrire les principes d'une planification stratégique qui inclut les orientations d'aménagement des territoires voisins. Les textes prévoient également, à ce chapitre, l'implication des associations locales d'usagers agréées, si elles en font la demande.

Le principe d'élargissement de l'association des acteurs parties-prenantes rejoint des objectifs de mobilisation et d'interaction au sein de ce collectif. Une des particularités de la loi SRU est la généralisation d'une concertation avec le public qui rend cette dernière obligatoire pour toute élaboration ou révision d'un SCoT ${ }^{3}$. L'article L. 300-2 du code de l'urbanisme précise ainsi que « le conseil municipal ou l'organe délibérant de l'établissement public de coopération intercommunale délibère sur les objectifs poursuivis et sur les modalités d'une concertation associant, pendant toute la durée de l'élaboration du projet, les habitants, les associations locales et les autres personnes concernées dont les représentants de la profession agricole ${ }^{4}$ ». Cependant, l'élaboration ou la révision d'un SCoT, portée par un EPCI ou un syndicat mixte, a la charge de préciser les modalités de cette concertation. En effet, si l'ouverture de la planification stratégique à un collectif d'acteurs plus varié est prévue par la loi SRU, celle-ci ne la réglemente pas davantage, laissant une grande marge de manœuvre aux acteurs porteurs du projet urbain. Plus encore, l'ouverture du processus, nommé concertation par le législateur, peut prendre plusieurs formes : réunions publiques, information, consultation, ou concertation. Elles dépendent en définitive d'une jurisprudence en la matière et des représentations locales de ce type de processus. En définitive, si la concertation définie comme l'association de personnes publiques, et plus largement la société civile, est obligatoire, les porteurs de projets (EPCI ou syndicat mixte) ne sont pas liés par les résultats de cette concertation ${ }^{5}$.

Ces dispositions montrent bien que plus qu'un résultat ou une production, c'est le processus de mobilisation sociale en lui-même qui prime et qui doit se pérenniser au-delà d'une opération ponctuelle ${ }^{6}$. En tant qu'instrument d'action publique, le SCoT instituerait de nouvelles formes d'action publique qui s'appuient sur le référentiel d'une nouvelle gouvernance.

\section{LE SCOT, Un INSTRUMENT DE GOUVERNANCE TERRITORIALE}

La gouvernance est «l'ensemble des nouvelles formes d'action publique qui permettent, sous le mode du partenariat, la négociation entre l’État, les collectivités territoriales, les secteurs économiques et associatifs, les groupes d'intérêt et la société civile» (Bertrand et Moquay, 2004). Les relations de coopération et de coordination développées entre acteurs issus des sphères institutionnelles, économiques et plus généralement privées ont pour finalité d'engager des dynamiques de projets et des actions mais aussi d'instituer des référents collectifs et des normes (Moquay, 2005). Appliquée à un territoire, «la gouvernance territoriale se caractérise précisément comme le processus, essentiellement dynamique, qui vise à la formulation et/ou la résolution d'un ou de problème(s) productif(s) le plus souvent inédit(s). [...] Le processus de gouvernance territoriale permet d'articuler entre eux les acteurs situés sur le même site géographique mais aussi dans le même temps, il relie les acteurs locaux et les niveaux macro-économiques globaux du national voire

2. Il s'agit principalement de l'habitat, de l'urbanisme, des déplacements, de l'aménagement ou de l'environnement.

3. La concertation était jusqu'alors encadrée par la loi du 18 juillet 1985 qui réservait ce dispositif aux opérations modifiant de façon importante le cadre de vie ou l'activité économique d'une commune.

4. Cet article concerne toute élaboration ou révision d'un document d'urbanisme, création d'une ZAC (zone d'aménagement concertée).

5. La procédure est complétée et légitimée à travers l'enquête publique. Alors que les anciens SDAU étaient simplement mis à disposition du public pour consultation a posteriori, l'approbation du SCoT, n'intervient in fine qu'à l'issue d'une enquête publique qui recueille les observations du public, des avis des communes, des personnes publiques consultées et du préfet.

6. Le SM ou l'EPCI maître d'ouvrage assure en effet non seulement l'élaboration d'un SCoT mais également son suivi et son évaluation. 
du supranational » (Pecqueur, 2001). C'est un processus qui réunit un collectif d'acteurs hétérogènes qui, en se mobilisant, poursuit des objectifs de construction de ressources et de production de normes d'action communes. Trois composantes structurent la gouvernance territoriale : une communauté d'intérêt, une communauté d'action et une mise en commun des représentations de la ressource partagée (Raymond, sous presse). La communauté d'intérêt est composée par l'ensemble des acteurs qui ont trouvé un intérêt à agir ensemble pour résoudre un problème. La communauté d'action concerne l'ensemble des acteurs à qui la communauté d'intérêt délègue le pouvoir d'agir en son nom. C'est elle qui a la maîtrise d'œuvre de la gestion de la ressource partagée. L'une et l'autre communauté ne rassemblent pas forcément les mêmes acteurs, même si elles sont souvent sécantes. La ressource partagée, le territoire, est l'objet autour duquel la communauté d'action va agir en mettant en commun les représentations des acteurs.

Les propriétés de la gouvernance territoriale spécifient un processus toujours singulier et fortement lié aux situations locales : les acteurs parties prenantes et leur diversité, les enjeux et les problèmes posés, les périmètres retenus pour la planification stratégique, la géopolitique locale et la distribution du pouvoir politique orientent les modalités de la gouvernance territoriale. Ainsi, les formes de la gouvernance instituées dans le cadre de communautés d'action et d'intérêt sont plurielles. Le système de gouvernance territoriale généré par les acteurs peut avoir un lien étroit ou plus déconnecté d'un système de démocratie locale (Biarez, 1998, 1999). Le système d'acteur peut être ouvert ou fermé, figé ou souple et les formes de la participation peuvent varier, depuis les arènes fermées sur un cercle de décideurs coordonnés par un ensemble de règles institutionnelles, aux forums, plus largement ouverts qui accueillent la société civile et les populations ellesmêmes, censées plus facilement discuter des règles existantes (Jobert, 1992). Les configurations des communautés d'intérêt et d'action permettent de comprendre les spécificités de la régulation au sein d'un territoire.

La législation relative à l'élaboration d'un SCoT institue bien une nouvelle place aux collectivités et aux acteurs locaux. La participation de nouveaux acteurs et la mise en place de démarches de concertation apparaissent ainsi comme les gages d'une meilleure démocratie mais également, comme les garants de la réussite de la conduite de l'action publique. Comme nous l'avons indiqué plus haut, si la dynamique d'ouverture des processus de gestion et de planification est engagée par la loi, les règles et les normes d'action, les modes de gouvernance associés sont l'œuvre de la décision locale des porteurs de projets. D’une manière générale, un SCoT s'élabore à partir de travaux de groupes ou de commissions de l'organisme porteur de la démarche, souvent étayés par des études complémentaires réalisées par des partenaires locaux, des experts ou des scientifiques. Selon une récente analyse (CERTU, 2008), deux modalités de gouvernance territoriale autour d'un SCoT existent. Soit elle respecte les textes législatifs et les acteurs sont associés essentiellement aux phases officielles de consultation et participent peu aux discussions amont. Les commissions de travail sont le lieu de débat et de décision mais pas de concertation. Les avis des acteurs locaux sont recueillis par un intermédiaire (souvent le maître d'œuvre) et ont ainsi une audience restreinte. Soit le mode de gouvernance correspond à une association plus large des acteurs locaux dans le cadre d'un dispositif de concertation, sans pour autant que les orientations dégagées ne soient prises en compte dans l'élaboration du SCoT. L'intégration plus ou moins forte de la procédure au processus de décision politique relève des contextes géopolitiques locaux et des configurations des territoires investis. Le SCoT est un instrument d'action publique qui a l'ambition d'articuler et de faire dialoguer un ensemble d'acteurs hétérogènes, suivant une mobilisation sociale autour d'une ressource commune. Il apparaît ainsi comme un instrument de gouvernance. Cependant, cette gouvernance reste à définir et est un lieu à construire et à investir.

\section{Le Pays du Grand Clermont : le triptyque Charte, Contrat, SCoT}

Dès la constitution du Pays du Grand Clermont (Puy de Dôme, Auvergne, France), le choix des acteurs s'est orienté vers l'élaboration d'un SCoT sur ce même périmètre (fig. 1). Le projet 
du pays et la planification stratégique du Grand Clermont se présentent comme deux processus concomitants. Le périmètre du Pays a été fixé à partir du zonage du schéma directeur de 1995. Le SCoT définit un espace d'action au même temps qu'il reconfigure et transforme un système d'acteurs pré-existant.

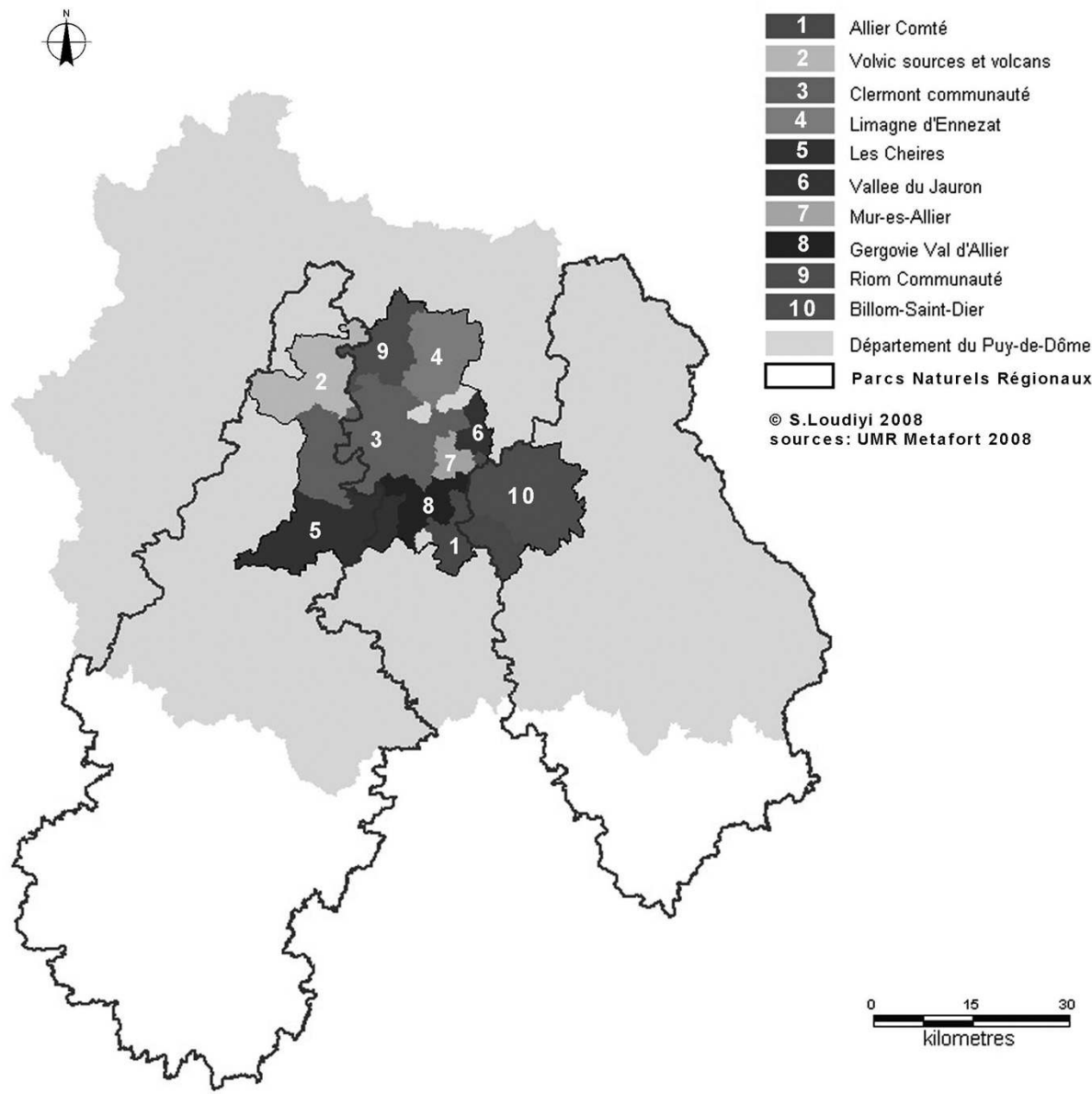

Figure 1 : Le Pays du Grand Clermont

The Grand Clermont area

Le périmètre du Pays du Grand Clermont couvre une surface de $1330 \mathrm{~km}^{2}$ avec 106 communes et compte environ 400000 habitants en $2002^{7}$. Le pays englobe une grande partie de l'aire urbaine (94\% de la population de l'aire urbaine de Clermont-Ferrand). La structure institutionnelle de pays se compose de dix intercommunalités (dont la communauté d'agglomération de Clermont Métropole) et de trois communes isolées. Situé au cœur de la région Auvergne autour de Clermont-Ferrand, le pays réunit des espaces urbains, périurbains et ruraux. Il s'étend au nord vers les terres de la Limagne productive, au sud vers les pays coupés, à l'ouest vers la Chaîne des Puys et englobe une partie des monts du Livradois à l'est. Le périmètre du pays est sécant avec deux territoires de projets, à l'ouest, le PNR des Volcans d'Auvergne, à l'est, le PNR du Livradois- 
Forez. De fait le territoire est fortement contrasté et les enjeux sont assez diversifiés selon que l'on se situe dans des espaces dits périurbains, urbains ou ruraux. Le défi auquel doit répondre le Pays et le SCoT est l'amélioration de l'attractivité démographique et économique de l'agglomération et de son rayonnement extérieur. Le Pays ambitionne d'atteindre le seuil des 500000 habitants à l'horizon 2025, « seuil à partir duquel, une agglomération peut prétendre à une envergure nationale voire internationale ${ }^{8} »$.

\section{UN ESPACE D’ACTION : DU SCHÉMA DIRECTEUR VERS UN SCHÉMA DE COHÉRENCE}

La généalogie de l'élaboration du SCoT s’inscrit dans la continuité de la charte et du contrat de Pays du Grand Clermont. La réflexion sur la mise en place du pays du Grand Clermont a été initiée dès 2001 par la communauté d'agglomération de Clermont Communauté, la communauté de communes de Riom Communauté et le Conseil Général du Puy-de-Dôme. Le projet a été animé par un syndicat mixte, le SEPAC (syndicat d'élaboration et de programmation de l'agglomération clermontoise). Il est une "réincarnation » du précédent syndicat mixte chargé de l'élaboration du schéma directeur de l'agglomération. Le SEPAC est saisi en 2002 pour l'élaboration du projet de Pays, dont le contrat sera signé en 2004. En 2006, il se mobilise pour la révision du schéma directeur de 1995, qui prendra la forme de l'élaboration d'un SCoT sur le périmètre du Pays. Les acteurs locaux visent ainsi « l'efficacité en évitant la multiplication des démarches, des échelles et des structures ${ }^{9} »$. Ces objectifs rejoignent le principe de la continuité de l'action publique et de l'organisme qui tient à jour les documents de planification, en somme la « mémoire » du processus de planification.

Les communes et les EPCI situés sur le périmètre du SD de 1995 sont invités dès l'origine à participer à la conception du futur Pays avec une possibilité d'adhésion au cours du processus des territoires limitrophes. À ce stade, le projet est conçu comme une démarche collective fondée sur l'adhésion des territoires à une vision partagée ${ }^{10}$. L'élargissement du périmètre du SD a pour vocation de tenir compte de l'évolution du territoire. Avec l'élargissement spatial, il y a une évolution du système d'action et des identités d'actions associées.

\section{LE SYSTÈME D’ACTEURS}

Le SEPAC représente l'instance qui assure le portage politique du SCoT, validant les grandes étapes de la démarche et la méthodologie utilisée. Il se présente comme « l'outil de réflexion prospective et d'organisation du Grand Clermont » et se définit comme « une instance de gouvernance, un espace de réflexion et de dialogue ${ }^{11}$ ». Les délégués des intercommunalités siégeant au sein du Comité Syndical du SEPAC décident des orientations du SCoT par délibération. Les décisions sont préparées par trois commissions d'élus ${ }^{12}$, par le bureau du SEPAC et par le comité de pilotage du SCoT. La gouvernance du SEPAC se décline ainsi à travers une communauté d'intérêt (le comité syndical représentant la population du Pays) et une communauté d'action (un bureau, des commissions, un comité de pilotage). La planification stratégique, à travers le SCoT, s'énonce

8. Communiqué de presse. Le Grand Clermont à la foire de Cournon, septembre 2008. « Faire du Grand Clermont une métropole européenne qui compte, doublée d'une locomotive régionale dynamique, implique de voir plus grand et plus loin. Notre territoire doit dès maintenant renforcer son attractivité pour relever ses défis démographique et économique en s'ouvrant sur l'extérieur » Dominique Adenot, actuel président du SEPAC (2008).

9. La lettre du Grand Clermont, Bulletin de liaison sur l'avancement de la démarche d'élaboration du projet du Grand Clermont, décembre 2002, n 4, 4 p.

10. Un modèle d'organisation future du territoire est proposé à l'amont et plaide pour une organisation polycentrique de l'espace clermontois. Faire partie du futur périmètre implique une adhésion à ce modèle d'organisation.

11. Allocution de Serge Godard, maire de Clermont-Ferrand, Président du SEPAC, le 13 janvier 2008 lors de l'ouverture de la réunion de restitution du SCoT.

12. Cadre de vie et qualité du territoire, développement économique et fonctions métropolitaines, solidarité et cohésion sociale. 
ainsi comme un projet essentiellement politique, piloté en conséquence par les élus locaux qui en ont l'initiative et qui en gardent la maîtrise jusqu'à son aboutissement.

L'agence d'urbanisme assure la maîtrise d'œuvre et l'assistance à maîtrise d'ouvrage. Les travaux des commissions d'élus sont étayés par des études et diagnostics réalisés par des "groupes thématiques techniques » qui réunissent une diversité d'acteurs : agence d'urbanisme, techniciens des intercommunalités, bureaux d'étude spécialisés, services de l'État, organismes gestionnaires, acteurs socio-économiques, etc. L'attente du SEPAC envers son maître d'œuvre se manifeste à travers trois points : " une écoute et une concertation avec les élus dans un esprit de solidarité », « une méthode et une qualité du travail », «le respect du calendrier et du coût du projet ${ }^{13}$ ». Le déroulement de la première phase d'élaboration du SCoT, «le diagnostic partagé », va ainsi être structuré et contraint par ce cadre.

\section{LA PARTICIPATION AUTOUR DU DIAGNOSTIC DU SCOT}

La mise en œuvre du «diagnostic partagé » constitue un moment d'ouverture du processus d'élaboration du SCoT du Grand Clermont. Cette ouverture correspond à un temps où les travaux des « commissions thématiques » et les positionnements politiques proposés par le SEPAC sont mis en débat entre un collectif d'acteurs privés et publics. Elle est structurée par trois opérations : l'information, la consultation et la concertation. Deux temps peuvent être identifiés : une phase amont où une enquête est réalisée et qui vise à recueillir « les attentes des acteurs » vis-à-vis du SCoT, puis la production d'un bilan du schéma directeur, et une phase, où sont organisés des débats autour de questions et enjeux du territoire désigné.

\section{Consultation et information}

La participation débute par un questionnaire visant à recueillir les « ressentis » envers le schéma directeur de 1995 et la charte ainsi que « les attentes des acteurs ${ }^{14}$ » vis-à-vis du SCoT. Cette phase s'apparente à une procédure de consultation où est recueilli l'avis des acteurs, essentiellement des élus politiques, sur la démarche initiée. Elle est considérée par le porteur du projet comme « une première étape de concertation avec les élus du Grand Clermont ». À travers ce questionnaire, le SEPAC « interpelle » ainsi les élus des EPCI, des personnes publiques associées et des membres du conseil de développement du Pays. Sont également organisées des « concertations délocalisées », pour expliquer les finalités du questionnaire et stimuler l'implication des acteurs dans la démarche ${ }^{15}$. Les résultats de cette phase révèlent que $60 \%$ des maires des communes ${ }^{16}$, 8 personnes publiques sur 10 associées et seulement $30 \%$ des conseillers communautaires ${ }^{17}$ ont répondu au questionnaire. Les territoires où l'on relève des taux de réponses proportionnellement importants, se situent principalement dans les espaces aux portes de l'agglomération ${ }^{18}$. Au niveau communal, les taux de réponses reflètent un gradient diminuant à mesure que l'on s'éloigne du centre de l'agglomération. Un degré d'implication fort semble concerner les élus des territoires où les enjeux d'urbanisation diffuse sont importants. On suppose aussi que le degré d'engagement à ce stade est plus fort au sein du système d'acteurs auparavant concerné par le schéma directeur.

La participation autour du SCoT se conçoit également comme une démarche d'information sur le bilan des actions passées. La diffusion du bilan du schéma directeur à la suite des résultats de l'enquête «attente des acteurs » permet de mettre en lien un savoir technique et le ressenti des

13. Extrait de l'allocution de Serge Godard, ibidem.

14. Questionnaire envoyé à l'ensemble des maires (106), aux délégués communautaires (315), aux personnes publiques associées et aux membres du conseil de développement qui doit représenter la société civile, juin-septembre 2006.

15. Attente des acteurs. Enquête $2^{\mathrm{e}}$ semestre 2006. Agence d'urbanisme Clermont Métropole. 58 p.

16. 69 réponses sur les 106 maires interrogés.

17. 97 réponses sur 315 délégués des communautés de communes et de la communauté d’agglomération composant le Pays.

18. À l'exemple des CC Gergovie Val d'Allier et CC Volvic Sources et Volcans : $44 \%$, Riom Communauté : 34 \%, Vallée du Jauron : $30 \%$. Ce sont des EPCI situés dans le champ de l'influence urbaine. 
acteurs. Le bilan met l'accent sur les enjeux de l'étalement urbain en désignant les territoires où le processus d'urbanisation a été important et en mettant en lumière les différentes formes urbaines produites en conséquence. Les références générales évoquées et l'aspatialité du phénomène du mitage urbain visent un consensus global préalable à un travail de fixation des limites et de localisation des activités et régulation des usages. Sa fonction d'information sur des enjeux d'intérêt général vise une mobilisation sociale pour le traitement de ces enjeux aussi bien qu'une légitimation a priori de l'ensemble des décisions qui seront mises en œuvre à travers la concertation.

\section{La concertation}

Le choix du maître d'œuvre de la méthode et des outils pour la conduite locale de la concertation puise ses fondements dans les attentes du maître d'ouvrage sur la qualité de la méthode utilisée, son respect du coût prévisionnel et des calendriers fixés. L'agence d'urbanisme met ainsi en place un outil opérationnalisant la conduite de la concertation : le « jeu de carte ${ }^{19}$. Cet outil est présenté aux acteurs participant aux débats comme innovant et inédit. Il vise à faciliter la construction d'un diagnostic partagé à partir d'un ensemble de questions, problèmes et enjeux soumis à l'interprétation des acteurs parties prenantes. Le jeu permet d'animer les séances de concertation et d'organiser l'interaction des acteurs.

Dans la mise en œuvre de cet outil, l'agence d'urbanisme s'est librement inspirée d'un outil existant et développé par l'équipe de recherche d'AgroParisTech ENGREF de Clermont-Ferrand. Notre proposition d'animer les débats à partir de la méthodologie du « Jeu de territoire 20 » été refusée par l'agence, puisque la géopolitique locale était peu propice à ce genre d'opération : les conflits politiques, l'instabilité du système d'acteurs, l'ampleur des enjeux à traiter, plaidaient pour une intervention exclusive de l'agence d'urbanisme. La participation de l'équipe de recherche s'est orientée vers une demande de la part de l'agence de réaliser une conférence lors de la séance introductive des débats. L'objectif assigné à notre participation était de montrer aux acteurs tout l'intérêt de se mobiliser dans une démarche de $\mathrm{SCoT}^{21}$. Elle nous permettait en retour d'assister aux séances des commissions en tant qu'observateurs extérieurs.

L'élaboration du « jeu de carte » par l'agence d'urbanisme poursuit plusieurs objectifs affichés et se manifeste sur trois plans : d'abord faciliter l'interaction entre acteurs et donner la parole aux participants qui s'expriment peu ou pas habituellement, permettant ainsi l'expression d'identités d'action. Ensuite, produire une synthèse problématisant les enjeux du territoire suivant un processus de co-construction du diagnostic. La confrontation d'arguments différents permettrait ainsi l'ajustement des points de vue et l'instauration de normes et de règles d'action. Enfin, l'outil doit contrecarrer les effets de «lassitude » constatée chez les acteurs, puisque l'élaboration du SCoT suit de peu celle de la charte et du contrat de Pays. Par conséquent, l'outil a pour fonction de faciliter une mobilisation sociale autour du projet suivant une logique interactionniste et qui vise à atteindre un consensus, stabiliser un collectif d'acteur, une identité et sa pérennisation.

La constitution des groupes de participants aux séances fait l'objet d'un double processus : l'invitation des personnes publiques associées que sont les représentants de l'État, les services déconcentrés, les représentants des parcs naturels régionaux, les chambres consulaires, ainsi que les représentants des territoires limitrophes; l'invitation et la cooptation des élus délégués communautaires des différents territoires de projets constituant le pays du Grand Clermont. Globalement, chaque commission thématique devait réunir mensuellement 30 à 40 personnes.

19. Terme consacré par le maître d'ouvrage de la technique utilisée.

20. Le jeu est un « jeu d'expression » : il vise à faire exprimer aux acteurs des points de vue pour converger vers une vision partagée du territoire. Le jeu, qui se base sur l'usage des représentations spatiales, favorise la dynamique d'interaction du groupe, en mettant les acteurs en position de collaborer. Il offre un cadre collectif de production de règles. Il met en scène les raisonnements des acteurs et facilite l'apprentissage collectif (Angeon et Lardon, 2003). C'est un dispositif d'accompagnement des acteurs dans la formulation de leur projet.

21. La conférence prend place au sein d'une série de trois conférences où le chercheur joue le rôle de l'expert. Richard Raymond, Espaces naturels et environnement. Enjeux et identités d'un territoire. Présentation consultable à l'adresse : [http://www.clermontmetropole.org/html/scotau.html]. 
Pour garantir la présence des participants, un système de suppléance a été mis en place. En tout, quatre séances, pour chaque commission thématique, ont rassemblé les élus du Grand Clermont et les personnes publiques associées pour débattre des problématiques territoriales, s'accorder sur les enjeux posés et les modalités de réponse à ces enjeux. Les acteurs locaux légitimes pour débattre et construire le diagnostic partagé sont les élus du territoire, les personnes publiques associées telles que définies par les textes législatifs. Le collectif d'acteurs est ainsi réuni par une dynamique de cooptation (Hermet, 2005).

L'outil devant organiser l'interaction, le «jeu de carte » est un ensemble de fiches rédigées (fig. 2) de format A5 synthétisant un ensemble d'informations structurées à travers de grandes thématiques. Les « cartes du jeu » se composent de trois types d'informations : un état des lieux, qui n'est pas censé être remis en question (fig. 2a), le positionnement politique qui traduit les choix déjà engagés dans le pays (fig. 2b), et les projets en cours de réalisation que le SCoT doit appuyer voire légitimer (fig. 2c). Dans la forme, aucune iconographie ni aucune représentation spatiale des informations ne figure sur les fiches distribuées. Le statut de ces fiches fait l'objet d'une hiérarchie : certaines sont prioritaires et d'autres mineures. Les «joueurs » doivent faire le choix des premières avant d'aborder les secondes. Le « jeu» est mis en œuvre dans le cadre de séances dédiées à trois commissions thématiques ${ }^{22}$. La composition du « jeu de carte » est différente selon l'objet des commissions et des thématiques spécifiques abordées dans chaque commission. À l'amont de chaque séance, le « groupe technique thématique » (GTT) prépare les livrets diagnostics ${ }^{23}$ relatifs à chaque question traitée et valide le contenu des cartes proposées au jeu d'interaction. Les livrets diagnostics et les informations contenues dans les « cartes du jeu » sont un ensemble de connaissances que les acteurs sont censés maîtriser ${ }^{24}$. La distribution des « cartes » du jeu est présentée comme aléatoire. Le nombre de « cartes » et de «joueurs » varie tout au long des séances et chaque participant peut recevoir entre une et trois cartes. Les séances sont animées par les élus référents de chaque commission thématique.

L'outil présente un caractère ludique qui se traduit notamment par l'usage de la terminologie propre au jeu («Dame de Cœur », «Joker», etc.). Comme certaines règles du jeu en général le stipulent, après distribution des «cartes », un joueur détenteur de « la dame de cœur », carte maîtresse de la séance introduisant la thématique générale, doit initier le jeu par l'énonciation des informations contenues dans la carte, en débattre et apporter des compléments, voire se positionner par rapport à l'ensemble. La relève doit se faire spontanément, en référence aux cartes détenues par les autres joueurs. On suppose ici un dialogue autour d'apports/contradictions sur les informations énoncées à chaque fois par un « joueur» qui s'exprime. On suppose aussi que le « jeu » va permettre aux acteurs présents et de manière toujours spontanée de se prononcer sur les questions/problèmes évoqués. Les interactions visent une finalité pour chaque séance : déterminer les « cartes » (traduisant des informations) qui «doivent être harmonisées » et énoncer un ensemble d'enjeux identifiés comme prioritaires, que le SCoT doit gérer et réguler. Les questions posées lors de chaque séance sont consignées sur une « feuille de route ${ }^{25}$ » (fig. 3) distribuée aux participants. Le contenu du débat pour chaque séance est consigné en temps réel sur un écran projeté dans la salle, afin de dégager rapidement les enjeux associés à la question traitée.

22. "Cadre de vie et qualité du territoire », «Développement économique et fonctions métropolitaines » et « Cohésion sociale et solidarité ». Le triptyque entre en résonnance avec les objectifs d'un SCoT respectant les principes de durabilité.

23. Les livrets diagnostics sont un résumé détaillé sur une question issue, soit de la phase diagnostic pour la constitution du projet de pays, soit une synthèse nouvelle réalisée par le « groupe technique thématique ». Ils sont de format A4 et leur taille peut atteindre une centaine de pages.

24. Ces documents font l'objet d'un envoi postal avant chaque séance pour permettre aux participants de se rompre à l'exercice avant d'y assister. Selon l'ordre du jour, plusieurs livrets peuvent être envoyés aux acteurs avant la tenue de la commission.

25. Qui contient par ailleurs, la liste des «cartes du jeu » selon leur typologie, la liste des participants à la commission thématiques, la liste des membres du groupe thématique technique qui ont contribué à élaborer les synthèses territoriales proposées. Les trois grandes questions sont mises en exergue sur la partie droite de la « feuille de route » avec des rubriques libres pour y répondre. 


\section{SCOT du Grand Clermont}

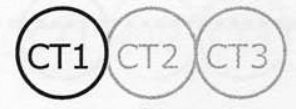

Carte « Etat des lieux 》

\section{Sensibilisation et protection contre les} risques naturels

\section{Développement de la culture du risque inondation}

Trois types de crues existent sur le territoire du Grand Clermont : les inondations de plaine (Allier), les crues torrentielles (rebord de faille) et les inondations urbaines (Clermont Ferrand). L'aléa inondation est bien réel.

Pour limiter l'exposition des populations aux risques, différents outils réglementaires ont été ou vont être mis en place comme les Plans de Prévention des Risques inondation (PPRi). Ces outils règlementaires doivent s'accompagner du développement d'une culture du risque et d'un réseau de vigilance comme le service d'annonce des crues mis en place par la DDE pour le Val d'Allier.

Les enjeux majeurs concernent aujourd'hui la gestion des crues torrentielles avec une réduction de la vulnérabilité s'imposant dans les choix urbains et un entretien des cours d'eaux.

\section{Connaissance et intégration du risque mouvement de terrains}

Le risque mouvement de terrain se décline sous plusieurs formes: mouvements dus au retrait gonflement des argiles, glissement de terrain, chutes de blocs et éboullements et séismes.

II n'existe cependant pas de document réglementaire précis, ni d'étude exhaustive. Certaines prescriptions s'imposent néanmoins aux constructions, notamment en prévention du risque de séisme.

Limiter l'exposition des usagers aux risques concernant la qualité de l'eau.

S'il n'existe pas de risques bactériologiques concernant la qualité de l'eau, il existe des problèmes d'arsenic et de plomb, conséquence de la nature physique des aquifères cristallins et volcaniques pour l'un et de vétusté de certaines canalisations pour l'autre.

Ces risques nécessitent un traitement préalable et une meilleure connaissance des réseaux d'adduction.

Figure 2 : Trois modèles de « cartes de jeu » distribuées

(a) carte " état des lieux » Protection et valorisation des milieux et ressources naturels.

Three models of the "playing cards"

(a) "statement" card; Protecting and valuing natural resources. 


\section{SCOT du Grand Clermont}

Carte « positionnement politique »

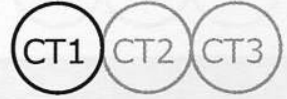

\section{Protéger la ressource en eau}

Acteur : Conseil général, SEPAC, communes et EPCI

Date : Charte de Pays du Grand Clermont (2004)

Les élus ont conscience que les ressources en eau du Grand Clermont sont abondantes et de bonne qualité, mais aussi fragiles et vulnérables. L'enjeu de la sécurisation des quantités et de la qualité d'eau disponible se pose à l'échelle du Grand Clermont. II figure dans la charte de pays du Grand Clermont et trouve des relais dans les politiques menées par les collectivités locales à travers les Contrats de restauration et d'entretien des rivières.

En ce qui concerne la ressource de la Chaîne des Puys, ses potentialités sont très élevées. C'est une ressource précieuse car très constante du fait de la régulation par le filtre volcanique. Mais elle se situe sur des terrains volcaniques avec une forte percolation à travers des sols très filtrants et est de ce fait très exposée aux pollutions.

La ressource de la nappe alluviale de l'Allier est plus importante (elle couvre $60 \%$ des besoins en eau potable du département) et c'est une zone stratégique pour l'agglomération clermontoise puisque la principale zone de captage se situe au niveau de Cournon, Mezel et Dallet. Les eaux pompées sont donc très vulnérables à la pollution des eaux de ruissellement, des traitements des cultures, des rejets non traités, des pollutions accidentelles sur les routes ...

Figure 2 : Trois modèles de « cartes de jeu » distribuées

(b) carte " positionnement politique » Protéger la ressource en eau. Three models of the "playing cards"

(b) "political positioning" card; Protecting water resources. 


\section{SCOT du Grand Clermont}

Carte « démarches, projets en cours »

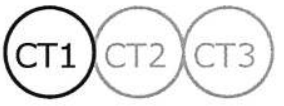

\section{Schéma départemental des carrières}

\section{Acteur : Etat / DRIRE}

Date : le schéma est en cours de révision et devrait être approuvé en 2007.

Le schéma départemental des carrières conduit une réflexion sur l'impact des carrières sur l'environnement mais aussi sur I'utilisation rationnelle et économe des matières premières minérales du département. Il doit être un document de référence proposant des orientations pour limiter l'impact des carrières sur l'environnement, tant au niveau de l'exploitation que de la remise en état des sites d'extraction.

Il doit éclairer la commission départementale des carrières sur toute demande d'autorisation de carrières.

Les grandes orientations du futur schéma sont :

Une orientation claire vers les roches massives et une diminution progressive des prélèvements de roches alluvionnaires

L'interdiction de toute nouvelle carrière d'alluvions récentes mais une autorisation sous conditions pour des carrières d'alluvions anciennes

Des propositions de reconversion des anciens sites

Des préconisations pour un usage économe des ressources et une sensibilisation des professionnels de la filière.

Figure 2 : Trois modèles de « cartes de jeu » distribuées

(c) carte « démarches, projets en cours "Schéma départemental des carrières.

Three models of the "playing cards"

(c) "measures, plans in progress" card; departement plan for quarries. 


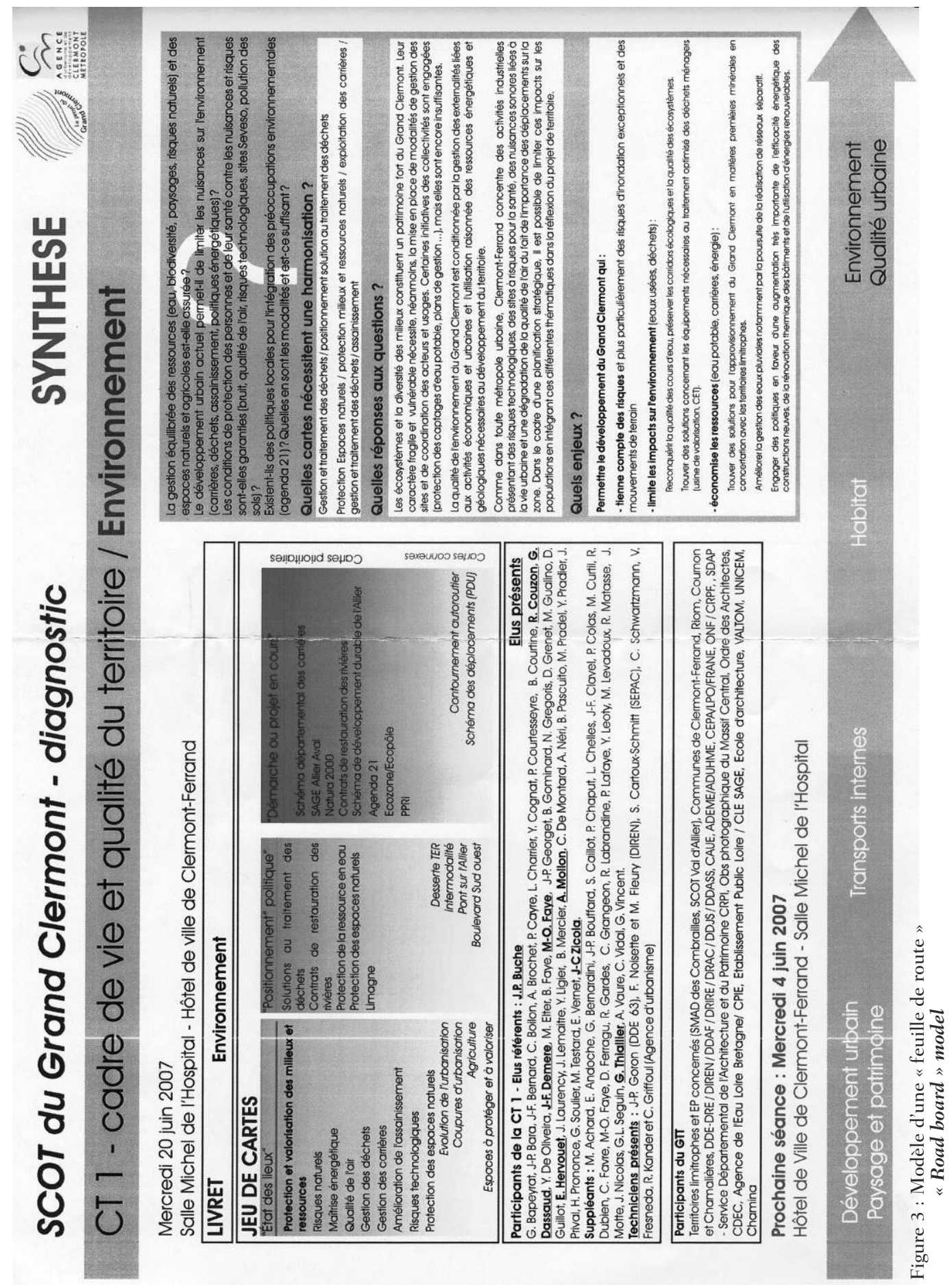


La mutualisation du produit final de l'ensemble des séances pour chaque commission thématique se fait dans le cadre d'une séance de synthèse, à travers une hiérarchisation des cinq enjeux prioritaires suivant une méthode de pondération permettant de donner un poids différencié pour chaque enjeu. Un calcul mathématique permet de faire émerger pour chaque groupe d'acteurs les principaux enjeux identifiés, qui constituent, de fait, les questions à traiter en priorité par le SCoT. La transversalité des enjeux identifiés est traitée en revanche en comité restreint par les élus référents et les techniciens de l'agence d'urbanisme. Les résultats des débats, la production d'un diagnostic partagé, font l'objet de séances de restitution auprès de l'ensemble des délégués communautaires des EPCI adhérentes et auprès de la société civile pour information.

\section{EFFETS PROPRES ET EFFETS INDUITS DU PROCESSUS DE PARTICIPATION AUTOUR DU DIAGNOSTIC DU SCOT}

La conduite locale de la concertation autour du diagnostic partagé du SCoT donne le primat à la mobilisation sociale, au processus d'interaction régissant les relations entre acteurs, à la construction d'un accord et enfin, à la production d'une vision partagée. Les séances ont globalement attiré peu d'élus (proportionnellement à ce qui était prévisible) et variablement selon les thématiques des commissions. La part des élus et des personnes publiques associées approchait plus souvent le tiers des participants "inscrits » aux différentes commissions. On constate néanmoins une tendance participative à la hausse pour les commissions abordant d'une part, le développement économique et d'autre part, la cohésion sociale et la solidarité. La qualité des acteurs présents trahit la surreprésentation d'élus communautaires de certains territoires de projets et de l'absence soutenue d'autres. Si l'objectif de ces séances de débat est de réunir la pluralité des acteurs de l'agglomération, certaines catégories des «personnes publiques associées » brillaient par leur absence, à l'instar des représentants des PNR ou encore des représentants de certaines chambres consulaires, alors que l'assiduité concernait le service déconcentré de l'État qui représentait dans ce processus, le préfet de région. Toutefois, dans la restitution des travaux des commissions, le porteur de projet déclare le diagnostic comme le fruit d'une large concertation, puisque 400 personnes ${ }^{26}$ y ont contribué. Le nombre est important et constitue une forme de légitimation du processus. Lecture a posteriori de la qualité du processus de mobilisation sociale autour d'une production partagée, le produit « diagnostic » incarne l’accord obtenu autour du projet.

La mobilisation autour de l'élaboration du diagnostic suit, grâce à l'outil « jeu de carte », une logique interactionniste. Les «cartes » structurent et ponctuent la séance; les acteurs se plient à l'exercice, se l'approprient au fil des séances et n’ont pas le temps de s'en extraire. Le déploiement discursif varie entre une lecture de la carte et des argumentaires construits. Le contenu des cartes distribuées fait généralement appel à des connaissances scientifiques (statistiques, sociologie, concepts et notions) qui permettent une interprétation causale des phénomènes auxquels est soumis le territoire. Ce contenu parfois très technique et par moment détaillé et complexe, est une occasion d'observer le déploiement de compétences discursives, dévoilant des identités d'actions en supplantant d'autres qui ne s'expriment pas forcement dans cette arène. Ainsi, peu de controverses ont lieu et lorsqu'elles émergent, sont vite résolues. On peut même avancer quà défaut d'accord explicite sur les questions/ problèmes évoqués, il n’y a pas de désaccord affirmé. La recherche absolue d'un consensus exclut de fait les sujets conflictuels et les acteurs et les groupes porteurs de ces sujets, sont souvent, dans le cas étudié, les moins compétents pour avoir voix au chapitre.

Le changement de la règle du « jeu » et des objectifs qui y sont assignés au cours du processus, est un effet induit par la forme instaurée de l'interaction. Devant la difficulté de certains acteurs à argumenter les informations qui sont en leur possession, la distribution des cartes n'est plus

26. Le nombre restitue la participation des acteurs à la phase dédiée à la concertation et ceux qui ont contribué, par les groupes techniques thématiques, à la préparation des matériaux utilisés dans le « jeu de carte ». 
aléatoire au bout de deux séances. De la même manière, face aux difficultés de certains acteurs à s'exprimer sur un sujet qu'ils ne maîtrisent pas, la faveur est donnée à un acteur « compétent » pour introduire la séance à travers la « carte maîtresse ». Et devant l'hésitation de certains à réagir spontanément, quelques élus référents animant les séances, prennent l'initiative d’interpeller les joueurs selon la carte détenue et qui est censée être « jouée ». Ainsi, la nécessité de produire une synthèse des enjeux identifiés à l'issue de chaque séance (et de contrecarrer le risque d'une absence de résultats) amène les techniciens de l'agence d'urbanisme à préparer celle-ci avant de la soumettre à l'approbation des élus référents. La synthèse est présentée comme une production in situ. Celle-ci n'est en revanche jamais révoquée par les acteurs parties-prenantes. L'objectif d'une construction partagée du diagnostic laisse ainsi place à la production d'un résultat, ici, fixé à l'avance. Cette production incarnerait la réussite du processus de concertation. Arriver à un accord apparaît, sous cet angle, plus important que le contenu de l'accord lui-même.

L'agence d'urbanisme avance lors d'un retour sur expérience à propos de la méthodologie employée, que les élus ne s'opposent pas au principe, que le contenu des interventions respecte l'ordre du jour, qu'il y a une implication active des participants avec une prise de parole de tous les élus et qu'enfin, il existe une prise de recul des élus lors de leurs interventions. Lors de la séance de synthèse, l'ensemble des participants présents «loue » les vertus du jeu dans sa facilitation de l'expression des acteurs. Le sentiment d'avoir contribué à l'émergence d'une volonté politique importe ainsi plus que d'avoir à subir son imposition (Pinson, 2004).

L'accord obtenu, à défaut de désaccord, et concrétisé à travers la production d'un diagnostic, permet à notre sens de faire émerger les premiers signaux relatifs à l'existence d'un collectif d'acteurs présent durant les phases de concertation. Les modes de sélection des acteurs sont certes particuliers et puisent dans les dynamiques de cooptation, mais la présence réitérée de certaines catégories d'acteurs permet de renforcer une dynamique d'échange. La diffusion des résultats auprès des EPCI à travers des réunions publiques ou auprès de la population à travers des expositions lors de foires, permet par ailleurs l'existence d'une identité politique, rend visible un territoire en affichant ses ambitions et dévoilant sa stratégie. La médiatisation et la valorisation des résultats participent aussi à l'implication sur le long terme, d'un système d'acteurs dans la poursuite des objectifs partagés. Bien entendu, ce collectif d'acteurs et l'identité politique du projet ne seront stabilisés in fine qu’à travers la poursuite de l'élaboration du SCoT.

\section{Conclusion}

La gouvernance territoriale comme instrument d'action publique s'opérationnalise à travers la mise en œuvre de démarches participatives où une pluralité d'acteurs, par leurs relations horizontales et non hiérarchisées, est censé apporter du changement et produire des choix dans la conduite de l'action publique. La mobilisation sociale et l'échange en sont les ingrédients. Néanmoins, le déploiement de la participation recouvre des démarches différentes allant de la simple consultation de la population à un objectif de co-production des décisions (Gaudin, 2007). Ces démarches restent ainsi marquées par une grande ambigüité sur les modalités de leurs conduites et leur finalité. La grande diversité des situations implique ainsi des interprétations contextuelles (Loudiyi et al., 2008). La prédominance des élus politiques et des administrations dans ces processus s'accorde mal avec les injonctions à la participation et à la démocratisation des prises de décision.

Les communautés d'intérêt structurant la gouvernance territoriale sont basées sur une démocratie représentative, où le poids des élus politiques est important et oriente la production des décisions. La communauté d'action peut permettre l'ouverture du processus d'interaction à une palette plus large d'acteurs, où le partage des visions, les ajustements mutuels peuvent produire des orientations nouvelles au sein du collectif d'acteurs. Cependant, le choix et la cooptation des acteurs jugés légitimes à participer, contribuent à escamoter l'émergence d'identités d'action nouvelles. La consultation apparaît ainsi comme un moyen de circonscrire la contestation possible dans ce type de processus, de même qu'elle implique une interprétation restrictive des besoins et 
attentes d'acteurs autres que les élus et les administrations. La conduite locale de la concertation, quant à elle, voile le caractère performatif d'une gouvernance qui peine à émerger. Si les liens horizontaux et non hiérarchisés entre acteurs permettent d'effacer les relations d'autorité, elles ne font cependant pas l'économie des relations de pouvoirs. La participation, selon ses modalités, est productrice de nouvelles élites. La somme n'est toutefois pas nulle, le sentiment de participer à la décision semble plus important qu'une réelle participation.

Les modalités de représentation de la ressource partagée comporte également des limites. Suivant les choix adoptés par les porteurs du projet, les références mobilisées peuvent être autant larges que précises. Nombre d'analyses ont démontré l'intérêt de l'usage des représentations spatiales comme objets médiateurs (Vinck, 1999) et comme support des interactions entre acteurs, réunis pour élaborer un projet de territoire (Lardon et al., 2001). Plusieurs méthodes d'appui et d'accompagnement des dynamiques d'acteurs ont été ainsi développées par des chercheurs pour la construction collective de diagnostic et de prospective des territoires. Le jeu y est présenté comme moyen de faire interagir des collectifs d'acteurs. L'action collective peut être ainsi initiée, accompagnée ou facilitée par le jeu (Angeon et Lardon, 2003, 2007; Lardon et al., 2008). Suivant les méthodes, le jeu peut s'appliquer soit à des territoires réels engageant des acteurs suivant leur rôle effectif, soit à des territoires fictifs où les acteurs jouent des rôles autres que les leurs. L'outil est soit « un jeu d'expression » (Lardon et al., 2008), soit un « jeu de rôles » (Etienne et Le Page, 2002; Bousquet et al., 2002). Dans tout les cas, des représentations spatiales (modélisations graphiques ou SIG) sont produites comme résultat des raisonnements spatiaux des acteurs sur des territoires à partir desquels ils énoncent des stratégies d'action. Dans le cas étudié, le « jeu de carte » s'apparente à un jeu de rôle dans la mesure où les identités d'action des acteurs et leurs territorialités sont écartées pour laisser place à des argumentaires construits a priori. L'objet de la concertation est bien un territoire réel mais qui est néanmoins labile. L'évocation d'un enjeu territorial mobilise chez les acteurs des objets géographiques porteurs de sens variés, qu'ils n'ont pas contribué à construire collectivement. Ceci induit des visions qui peuvent rester vagues et indéterminées. Les argumentaires construits à l'amont par le porteur de projet deviennent ainsi la seule solution à retenir. La recherche d'un consensus légitimant les décisions politiques, plaide pour une utilisation restrictive de références spatiales, pourtant au centre de la construction partagée du territoire. Cette recherche de consensus écarte ainsi les vertus d'un apprentissage des connaissances spatiales des uns et des autres, leur mutualisation et l'ajustement des visions portées par un ensemble d'acteurs aux identités d'action aussi variées qu'un territoire peut être contrasté dans sa structure et sa dynamique.

Les méthodes et outils de négociations et de concertation sont au centre d'enjeux sociétaux dans lesquels le chercheur est pleinement mobilisé. Le chercheur est vu comme un acteur qui peut jouer plusieurs rôles : celui de l'expert, du scientifique, de l'animateur, etc. Dans certaines situations, son rôle peut être assez ambigu. Dans le suivi du diagnostic du SCoT, notre rôle explicite était celui d'observateurs extérieurs, en même temps que notre compétence en matière de conception d'outils participatifs était reconnue sans pour autant qu'on ait pu intervenir dans la mise en place du « jeu de carte». La conduite locale de la concertation pour le diagnostic du SCoT du Grand Clermont, vue à travers sa mise en œuvre, montre dans quelle mesure des outils conçus par les chercheurs peuvent leur échapper et faire l'objet d'une appropriation « libre » de la part des acteurs. L'observation du dispositif nous permet de produire une analyse critique de sa mise en œuvre mais nous engage vers de nouvelles questions : qui a le contrôle de l'usage des outils produits? Comment les chercheurs peuvent-ils travailler l'appropriation des outils participatifs et leurs modalités d'usage? Comment clarifier le mandat du chercheur investi au sein d'une démarche participative? L'importance de la conception de méthodologies d'appui et d'accompagnement de démarches participatives nous semble aller de pair avec la nécessité d'engager une analyse réflexive sur la place du chercheur au sein de ces dispositifs. 


\section{Bibliographie}

Angeon (V.), LaRdon (S.), 2003.- « Dessiner et comprendre le territoire : quand le jeu devient un processus collectif d'apprentissage et de création ", dans Debarbieux (B.), Lardon (S.) (dir.), Figures du projet territorial, La Tour-d'Aigues, Éditions de l'Aube/DATAR, coll. «Bibliothèque des territoires », 270 p.

AngeOn (V.), LARDOn (S.), 2007. - « Le jeu de territoire : une méthode d'animation, un outil de gouvernance », Communication au Joint Congress of the European Regional Science Association (47th Congress) and ASRDLF (Association de Science Régionale de Langue Française, 44th Congress), Paris, 29 août2 septembre.

Bertrand (N.), Moquay (P.), 2004. - « La gouvernance locale, un retour à la proximité », Économie rurale, $\mathrm{n}^{\circ} 280$, p. $77-95$.

Biarez (S.), 1998. - « Sphère locale et espace public », Lien social et politiques, n 39, p. 127-138.

BiareZ (S.), 1999. - Le pouvoir local, Paris, Economica, 309 p.

Bousquet (F.), Barreteau (O.), D’aquino (P.), Etienne (M.), Boissau (S.), Aubert (S.), Le Page (C.), Babin (D.), Castella (J.-C.), 2002. - « Multi-agent systems and role games : collective learning processes for ecosystem management », dans JANSSEN (M. A.) (dir.), Complexity and ecosystem management: The theory and practice of multi-agent approaches, Cheltenham, Edward Elgar Publishers, p. 248-285.

Callon (M.), Lascoumes (P.), Barthes (Y.), 2001. - Agir dans un monde incertain. Essai sur la démocratie technique, Paris, Seuil, 400 p.

CERTU, 2008. - Prendre en compte l'agriculture et ses espaces dans les SCOT, Éditions du CERTU, 146 p.

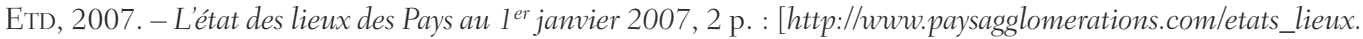
$p h p]$.

Etienne (M.), Le Page (C.), 2002. - « Modéliser les dynamiques paysagères pour accompagner un projet d’aménagement du territoire : le Causse Méjean », dans Actes du colloque Gérer les paysages de montagne pour un développement concerté et durable, Florac, Éditions du CEP, CD-Rom.

FERGUSSON (Y.), 2008. - « Réflexion autour des nouveaux instruments de la production urbaine : vers la fin du modèle keynésien? ", Métropoles, n 4, 28 p.

Gaudin (J.-P.), 2007. - La démocratie participative, Paris, Armand Colin, 125 p.

Helluin (J.-J.), 2005. - "Quels sont les effets de la "règle des 15 km" sur la maîtrise de l'étalement urbain en France? ", Les villes au défi du développement durable: Quelle maîtrise de l'étalement urbain et des ségrégations associées?, Colloque International du 24-25 novembre 2005, Sfax, Tunisie.

Hermet (G.), 2005. - « La gouvernance serait-elle le nom de l'après-démocratie? », dans Hermet (G.), Kazancigil (A.), Prud'homme (J.-F.) (dir.), La gouvernance. Un concept et ses applications, Paris, éditions Karthala, p. 17-47.

Jobert (B.), 1992. - «Représentations sociales, controverses et débats dans la conduite des politiques publiques », Revue française de science politique, vol. 42, n 2, p. 219-234.

Lardon (S.), Caron (C.), Bronner (M.-C.), Giacomel (G.), Raymond (R.), Brau (F.), 2008. - « Jeu de construction de territoire. Usage des représentations spatiales dans une démarche participative », Revue Internationale de Géomatique, Vol. 18, n 4, p. 507-530.

Lardon (S.), Moguay (P.), Poss (Y.), 2007. - Développement territorial et diagnostic prospectif. Réflexions autour du viaduc de Millau, La Tour-d'Aigues, Éditions de l'Aube, 377 p.

Lascoumes (P.), Le Gales (P.), 2004. - Gouverner par les instruments, Paris, Les presses de Sciences Po, $366 \mathrm{p}$.

Loudiy (S.), Bryant (C.), Laurens (L.) (dir.), 2008. - Territoires périurbains et gouvernance. Perspectives de recherche, Éditions du Laboratoire développement durable et dynamiques territoriales, Université de Montréal, 168 p.

Moquay (P.), 2005. - « L'État territorialisé, ou l'arroseur arrosé : les ambiguïtés de l'État face aux recompositions territoriales en France », dans Bherer (L.), Coldin (J.-P.), Kerrouche (E.), Palard (J.) (dir.), Le gouvernement des territoires au Québec et en France, Sainte-Foy (Qc), PUL, p. 85-116.

Pecqueur (B.), 2001. - «Gouvernance et régulation : un retour sur la nature du territoire », Géographie, Économie, Société, vol. 3, n² 2, p. 229-245. 
Pinson (G.), 2004. - « Le projet urbain comme instrument d'action publique », dans Lascoumes (P.), Le Gales (P.) (dir.), Gouverner par les instruments, Paris, Les presses de Sciences Po, p. 199-233.

RAYMOND (R.), 2007. - «Accords et controverses autour de la notion de gouvernance territoriale. Retours bibliographiques sur une notion à la mode ", Communication au Joint Congress of the European Regional Science Association (47th Congress) and ASRDLF (Association de Science Régionale de Langue Française, 44th Congress), Paris, 29 août-2 septembre.

RAYMOND (R.), (sous presse). - «Agreements and controversies around the notion of territorial governance. A bibliographic review of a fashionable notion ", dans Rey-Valette (H.), Lardon (S.), Chia (E.) (dir.), Governance: Institutional and learnig plans facilitating the appropriation of sustainable development, Special issue of International Journal of Sustainable Development, vol. 11, n 2/3.

VINCK (D.) (dir.), 1999. - Ingénieurs au quotidien. Ethnographie de l'activité de conception et d'innovation, Grenoble, PUG, 232 p.

Cet article a été reçu le 10 juin 2008 et définitivement accepté le 18 janvier 2009. 